\title{
Dynamically locked intramedullary interlocking nail for fracture shaft of tibia: An effective surgical protocol with minimal complications
}

\author{
Ramachandra N Badami ${ }^{1}$, Swetha Purohit ${ }^{2 *}$ \\ ${ }^{1}$ Senior Resident, ${ }^{2}$ Assistant Professor, ${ }^{1}$ Dept. of Othopaedics, Shimoga Institute of Medical Sciences, Shimoga, ${ }^{2}$ Dept. of \\ Anaesthesia, Subbaiah Institute of Medical Sciences, Shimoga, Karnatka, India
}

*Corresponding Author:

Email: swethapurohit@yahoo.com

\begin{abstract}
Introduction: Fracture shaft of tibia is one of the most common injuries presenting to a trauma center. Closed intramedullary interlocking nail is the gold standard surgical procedure. But even with advanced techniques there are several reports of delayed and nonunion of these fractures. We evaluated the results of dynamically locked tibia IMIL nail for fracture shaft of tibia.

Materials and Methods: 90 patients with fracture shaft of tibia were included in the study between 2013 to 2015 . AO type A1 and A2 were included in the study. Comminuted fractures, open injuries were excluded from the study. Outcomes were evaluated based on time to fracture union, limb alignment, walking ability and range of motion.

Results: There was fracture union in $100 \%$ of the cases an average duration of 16 weeks. There were no cases of nonunion/delayed union, significant mal-alignment of the fracture. The mean range of motion score was 3.6 and the mean walking ability score was 3.8 .

Discussion: Non comminuted fracture shaft of tibia treated with dynamically locked IMIL nail gave good to excellent results in 87 of the 90 cases with respect to fracture union, limb alignment, range of motion and walking ability. There were no cases with implant failure or limb length discrepancy. It produces good fracture compression and early weight bearing adds to its advantage. Conclusion: Dynamically locked IMIL nail for non comminuted fracture shaft of tibia gives excellent clinical and radiological outcome. It also avoids any chances of delayed/nonunion of the fractures of tibial shaft.
\end{abstract}

Keywords: Tibia, Shaft, Fracture, Dynamic, Locking, Nail.

\section{Introduction}

Fracture shaft of tibia has increased due to a large number of road traffic accidents happening in the modern day. The various treatment options available for the fracture shaft of tibia are: non surgical treatment, open reduction and plating, closed reduction and intramedullary interlocking nail (IMIL). ${ }^{1}$ Conservative treatment results in high chances of nonunion/malunion, stiffness of knee and ankle and delayed rehabilitation. Open reduction of the fracture causes extensive periosteal stripping, loss of fracture hematoma, high chances of infection and so on. Whereas, surgical treatment with IMIL nail tibia reduces all the complications and hence is the treatment of choice in the current practice.

The goal of fracture treatment is to establish as soon as possible the integrity of the bone and to start physical therapy. ${ }^{2}$ The outcome of each fracture healing depend on the performance and quality of surgery. Accordingly, each of the osteosynthesis implants has its advantages and disadvantages in relation to three basic problems of bone healing- Infection, Instability and Circulation. ${ }^{3}$ Low incidence of infection, high stability and strength of fragments of contact, the possibility of early mobilization of the patient while preserving soft structure and peripheral circulation, are a guarantee of success and quality of healing of fractures of the femur and tibia, treated using static and dynamic intramedullary osteosynthesis. ${ }^{4}$

Tibia IMIL nail has both static and dynamic locking options. Constant dilemma in modern orthopedic surgery is what type of intramedullary osteosynthesis to use static or dynamic. The common teaching is to use static IMIL nail for the fracture of shaft of tibia. Few studies have shown better results with static IMIL nail for Shaft of tibia fractures. ${ }^{4,5}$

We evaluated our results of dynamically locked tibia IMIL nail for the fractures of mid shaft of tibia.

\section{Materials and Methods}

We studied 90 patients of fracture shaft of tibia between June 2013 to June 2015. Fractures with AO type 3.2.A1 and 3.2.A2 were included in this study. Open fractures of tibia were excluded from the study. Also, bilateral tibia fractures and cases with associated injury to other parts which would impede the final outcome were excluded from the study. There were 38 cases of A1 fractures and 52 cases of A2 type fractures. All cases were operated within 48 hours of admission after obtaining a written consent for surgery.

Patients were operated on the fracture table keeping the knee in 90 degree flexion with a calcaneal pin attached to the fracture table. After painting and draping, incision taken over the midline from inferior pole of patella to tibial tuberosity. Patellar tendon split in the midline. Entry point taken about $2 \mathrm{~cm}$ below the joint line in the midline. Guide wire passed across the fracture site after obtaining the proper reduction by closed means. Serial reaming done and then appropriate size IMIL nail inserted. Two distal medio-lateral locking done under c-arm guidance. Any distraction at 
the fracture site closed by back striking and then the dynamic proximal locking alone was done.

Post-operatively patients were started with knee bending, quadriceps strengthening exercises from from day one and started with non weight bearing mobilization from day two for the first 2 weeks. Then, partial weight bearing mobilization for next 2 months. Patients were followed up at monthly for first 4 months and then 3 monthly till 1year. The average follow up was 15 months with a minimum follow up duration of 9 months.

At each follow up patients were assessed clinically for range of motion, signs of infection, walking ability, clinical alignment of the limb. Walking ability was assessed as per walking ability score Table 1. Range of motion at knee and ankle were assessed as per Table 2 .

Table 1: Walking ability scores

\begin{tabular}{|l|c|}
\hline Walking Ability & Score \\
\hline No Walking Possible & 0 \\
\hline Only with 2 Crutches or Frame & 1 \\
\hline $\begin{array}{l}\text { Walks with one cane but } \\
\text { marked limp }\end{array}$ & 2 \\
\hline $\begin{array}{l}\text { Walks with cane but noticeable } \\
\text { limp }\end{array}$ & 3 \\
\hline Normal & 4 \\
\hline
\end{tabular}

Table 2: Range of motion score

\begin{tabular}{|l|c|}
\hline $\begin{array}{l}\text { Range of Motion (Knee and } \\
\text { Ankle) }\end{array}$ & Score \\
\hline $\begin{array}{l}>20 \% \text { of stiffness as compared } \\
\text { to normal side }\end{array}$ & 1 \\
\hline $\begin{array}{l}10-20 \% \text { stiffness as compared } \\
\text { to normal side }\end{array}$ & 2 \\
\hline $\begin{array}{l}<10 \% \text { stiffness as compared to } \\
\text { normal side }\end{array}$ & 3 \\
\hline Normal- Equal to opposite side & 4 \\
\hline
\end{tabular}

Radiologically patients were assessed for fracture union based on bridging callus in AP and lateral views, any mal-alignment of the fracture site. Radiologically the fracture was said to be united when three of the four corticies were found to be united or in continuity with each other across the fracture site. Angulation at the fracture was measured as an angle between long axis of the proximal fragment and the long axis of the distal fragment.

\section{Results}

90 patients who met the inclusion criteria were included in the study. The mean age of the patients in the study was 36yrs (19-65 years). Road traffic accident was the most common mode of injury. The average surgical time was $40 \mathrm{~min}$. At an average follow up of 15 months there was union in $100 \%$ of the cases. Time to union ranged from 12 to $20 \mathrm{wks}$. There were no cases of nonunion or delayed union in our study population.
The average range of motion score was 3.6 [Fig-1]. The mean walking ability score was 3.8. [Fig-2]

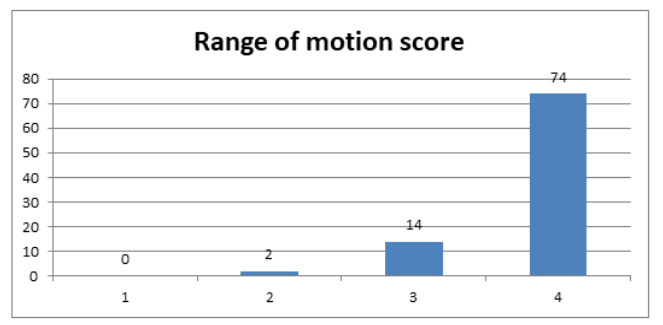

Fig. 1: Range of motion score

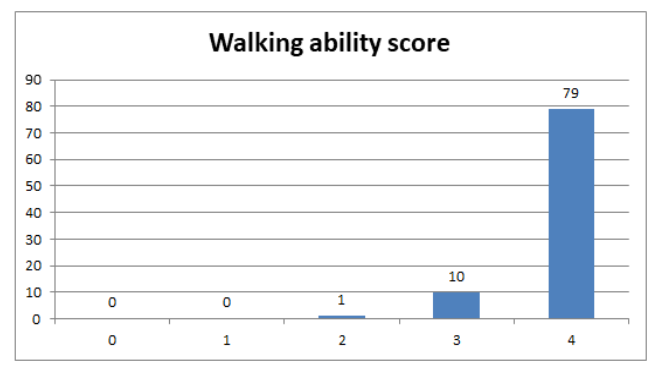

Fig. 2: Walking ability score

There were 4 cases with a varus angulation and 3 with valgus angulation at the fracture site which were less than 5 degrees. The angulations were noted at the immediate post-operative radiograph and did not show any increase in the angulation at the subsequent follow ups, till the fracture union. But there were no cases with clinical angulation at the fracture site as measured at the immediate post-operative period, subsequent follow ups till complete fracture union. There were no cases with rotational mal-alignment or any limb length discrepancy.

\section{Discussion}

The fracture shaft of tibia is a very common injury reported to a trauma center. It is most often treated by IMIL nailing which uses two proximal and two distal locking bolts (both static and dynamic locking bolts proximally). In case of any radiological signs of delayed union dynamisation of the nail is done by removal of the static locking bolt from the longer segment and making the patient walk full weight bearing. ${ }^{6}$ Despite improved treatment and union rates problems with delayed union and nonunion continue to occur. Although the treatment algorithm differs in each case, nail dynamization can be a quick, cost-saving, and effective method to promote healing. ${ }^{7}$ In our study of 90 patients with fracture shaft of tibia we used tibia IMIL we used only a dynamic proximal locking bolt with an early weight bearing mobilization. This protocol of ours gave an excellent result with a $100 \%$ union at an average of 16 wks. There were cases of delayed union or non union reported in our study. This is probably because of the compressive forces at the fracture site due to the dynamic locking and early 
weight bearing mobilization, at the same time not compromising the stability at the fracture site by the snugly fitting nail with 2 distal and 1 proximal locking bolt. Due to which there were no cases with significant (>5 degree) primary or secondary malunion or any rotational mal-alignment. This protocol also has an advantage of early mobilization and hence produced good to excellent results in all patients except two in this study. Similarly all patients except one had good to excellent results with respect to walking ability of the patient.

There were many studies which supported the use of dynamic IMIL nail in tibial shaft fractures. ${ }^{8-11}$ Josh Vaughn et al. ${ }^{10}$ showed a faster union in dynamic group than the static group, wherein an additional dynamisation procedure was needed in few cases to achieve union. Hernández-Vaquero D et al., also showed similar results of better union with dynamization, but their results did not show statistical significance. ${ }^{11}$

We had 2 patients with superficial infection, one of whom was a diabetic patient. Both resolved with intravenous antibiotics. 3 patients had anterior knee pain even after 1 year of surgery. This pain was probably related to the entry site of the nail along with patellar tendon splitting. There were no cases of implant failure or hardware breakage.

The main limitation of our study was that it did not compare static and dynamically locked IMIL nails in tibia. Also the study was limited to AO type A1 and A2 fractures and hence the results cannot be generalized to all tibial shaft fractures, specially more comminuted ones.

\section{Conclusion}

The tibia IMIL nail fixation is the gold standard for any tibial shaft fractures. In AO type A1 and A2 the dynamically locked IMIL nail gives excellent clinical and radiological outcome and rules out the chances of delayed or nonunion of the fracture. At the same time it doesn't compromise on the stability of the fracture. But the same cannot be generalized to all tibial shaft fractures and further studies are required to evaluate the outcome of the dynamically locked IMIL nail in comminuted tibial shaft fractures.

\section{References}

1. Labronici PJ, Franco JS, Silva AF, Cabral FMP, Soares MS, Toledo PRBL et al. Treatment of distal fractures of the tibia, Acta Ortop Bras. 2009;17(1):40- 5.

2. Eknath Pawar D, Saurabh Agrawal R, Atul Patil W, Sandeep Choudhary, Ghaniuzzoha Asadi. A Comparative Study of Intramedullary Interlocking Nail and Locking Plate Fixation in the Management of Extra Articular Distal Tibial Fractures. Journal of Evolution of Medical and Dental Sciences. 2014; 3(24)16;6812-6826.

3. Augustin G. Termičko oštećenje kosti tijekom operativnog zahvata: Magistarski rad. Medicinski fakultet Sveučilišta u Zagrebu, 2004.
4. Djemil Omerovic, Faruk Lazovic, Amel Hadzimehmedagic Static or Dynamic Intramedullary Nailing of Femur and Tibia Med Arh. 2015 Apr; 69(2): 110-113.

5. Dr. Ashish M Somani et al., Comparative study of static versus dynamic intramedullary nailing of tibia, International Journal of Orthopaedics Sciences 2017;3(3):283-286.

6. Omerovic D, Lazovic F, Hadzimehmedagic A. Static or dynamic intramedullary nailing of femur and tibia. Medical Archives. 2015; 69(2):110.

7. Vaughn J, Gotha H, Cohen E, Fantry AJ, Feller RJ, Van Meter $\mathrm{J}$ et al. Nail Dynamization for Delayed Union and Nonunion in Femur and Tibia Fractures. Orthopedics. 2016;29;39(6):e1117-23.

8. Basumallick MN, Bandopadhyay A. Effect of dynamization in open interlocking nailing of femoral fractures: a prospective random- ized comparative study of 50 cases with a 2-year follow-up. Acta Orthop Belg. 2002;68(1):42-48

9. Wu CC. The effect of dynamization on slow- ing the healing of femur shaft fractures after interlocking nailing. J Trauma.1997;43(2):263-267.

10. Vaughn J, Gotha H, Cohen E, Fantry AJ, Feller RJ, Van Meter $\mathrm{J}$ et al. Nail Dynamization for Delayed Union and Nonunion in Femur and Tibia Fractures. Orthopedics. 2016; 29;39(6):e1117-23.

11. Hernández-Vaquero D, Suárez-Vázquez A, IglesiasFernández S, García-García J, Cervero-Suárez J. Dynamisation and early weight-bearing in tibial reamed intramedullary nailing: its safety and effect on fracture union. Injury. 2012; 31;43:S63-7. 